\title{
El proyecto visado arquitectónico: descripción, caracterización y normalización documental
}

\author{
Por Enrique Orduña-Malea
}

\begin{abstract}
Resumen: La documentación arquitectónica es clave para conocer nuestra herencia cultural, pero su heterogeneidad y complejidad provoca problemas en su adecuada descripción y posterior recuperación en bases de datos. Este trabajo se centra en un tipo concreto dentro de esta clase de documentación: el proyecto visado. En primer lugar se procede a caracterizarlo documentalmente definiendo, describiendo y analizando sus partes constitutivas en función de las disposiciones legales y normas dispersas que lo regulan. A continuación se presenta un diseño conceptual que sirva como punto de partida para la construcción de una base de datos de proyectos. Finalmente se propone el Inpav (Identificador nacional de proyectos arquitectónicos visados), como estándar de identificación.
\end{abstract}

Palabras clave: Proyecto arquitectónico, Proyecto visado, Documentación arquitectónica, Diseño conceptual, Normalización, España, Inpav

Title: The architectural visa project: documental description, characterization and normalization

\begin{abstract}
Architectural documentation is a key element of knowing our cultural heritage. The heterogeneity and complexity of this documentation causes problems in their proper description and subsequent retrieval in databases. This work focuses on a specific typology within this type of documentation: the visa project. First, this typology is characterized as a document, defining, describing and analyzing its constituent parts according to the variety of laws and standards that govern it. Second, a conceptual design is proposed as a basis for construction of a project database system. Finally, the Inpav (National identifier of visa architectural projects) is suggested as standard identification.
\end{abstract}

Keywords: Architectural project, Visa project, Architectural documentation, Conceptual design, Normalization, Spain, Inpav.

Orduña-Malea, Enrique. "El proyecto visado arquitectónico: descripción, caracterización y normalización documental". El profesional de la información, 2009, marzo-abril, v. 18, n. 2, pp. 202-209.

DOI: 10.3145/epi.2009.mar.10

\section{Introducción}

La documentación arquitectónica es un elemento clave de nuestra herencia cultural (Blanco, 2004), pues refleja con inigualable exactitud cómo han sido creados, diseñados o modificados los lugares donde vivimos, constituyéndose por tanto en un verdadero testimonio del crecimiento, desarrollo y evolución de una ciudad.

Esta documentación constituye en algunos casos el único registro existente de primitivas estructuras así como de proyectos que nunca fueron construidos.

Con el progresivo crecimiento y regulación de la profesión, los arquitectos comienzan a trabajar en equipos donde el trabajo se distribuye entre distintos especialistas (ingenieros, arquitectos, diseñadores, etc.), provocando que el tiempo que transcurre entre el diseño y la construcción de una obra sea cada vez más pequeño (Ica, 2000), y que su producción documental no pare de crecer.

Según Hildesheimer (1987), los documentos de un archivo de arquitectura pueden dividirse en:

- Los que marcan las diversas etapas de elaboración del proyecto.

- Los que se elaboran en la etapa de realización.

- Los que consignan el estado de una obra realizada.

La documentación se crea siguiendo el curso de realización de una obra, agrupándola generalmente en series por arquitecto o por obra.

La descripción tiende a hacerse mediante la ISAD (G) aunque, según el Ica (Maygene, 2000), dentro de ella se pretende elaborar un estándar internacional para la descripción archivística de registros arquitectónicos. 
Pese a todo la descripción es complicada debido, entre otros, a los siguientes factores:

- Heterogeneidad de documentos (planos, correspondencia, maquetas, etc.).

- Diferencias entre países de las instituciones de archivo, la profesión de arquitecto y la reglamentación de construcción.

- Diferencias entre países de las características constructivas y de los materiales (Nyambayo; Faraj; Amor, 2000; Ekholm, 2008).

- Distintas necesidades de información según se trate de un archivo colegial (documentación de distintas fuentes), de un estudio (quizá un solo arquitecto firmante), etc.

Este trabajo se centra en un tipo concreto dentro de la documentación arquitectónica: el proyecto visado por un colegio profesional.

La bibliografía al respecto es escasa, destacando el trabajo de Carrascal-Simón (1994), centrado en el estudio de las fases de un proyecto, las distintas normativas de los colegios y la legislación pertinente.

El tratamiento documental es complejo, pues el proyecto visado se encuentra a mitad de camino entre el mundo bibliotecario y el archivístico.

Pese a que su ordenación física requiere de los principios del orden natural y procedencia (el conjunto de proyectos de una obra forma una serie documental), su descripción mediante $I S A D(G)$ no parece la más adecuada.

Las carencias en la descripción provocan problemas de recuperación en las bases de datos debido a limitaciones y errores conceptuales, lo que genera dificultades para explotar adecuadamente esta información.

Esto implica un problema en los estudios de arquitectura, donde los proyectos visados son fuentes de información básicas para conocer cómo se ha abordado anteriormente un contratiempo, qué materiales se han usado, etc. Es decir, gestión del conocimiento.

Ante esta situación, los objetivos de este trabajo son:

- Analizar el proyecto visado arquitectónico en España.

- Proponer un modelo conceptual que sirva tanto para elaborar una base de datos de proyectos como para facilitar el control de éstos.

- Proponer el código Inpav (Identificación nacional de un proyecto arquitectónico visado).

El estudio de estos parámetros constituye la base para una futura especificación de los distintos campos de recuperación (atributos de las entidades), así como para la normalización de los mismos, lo que conllevaría la creación de un modelo completo preparado para el diseño de unas reglas de catalogación enfocadas a este tipo documental, aspecto que se sale de los propósitos de este trabajo.

\section{El proyecto visado como tipo documental}

Un proyecto visado "es el conjunto de documentos mediante los cuales se define la obra, se determinan y justifican técnicamente las soluciones adoptadas, de acuerdo con las especificaciones requeridas por la normativa aplicable y las necesidades previstas para el edificio" (Ley 3/2004, Comunidad Valenciana).

\section{"El proyecto visado se puede analizar en dos niveles: según su tipo y según su composición"}

Siguiendo a Carrascal y Gil (2008), un proyecto arquitectónico visado cumple las siguientes funciones durante su ciclo de vida:

- Valor técnico: es una herramienta de trabajo para que terceros, con los conocimientos necesarios, puedan entender y ejecutar lo plasmado en el proyecto.

- Valor legal: es una parte del proceso administrativo que permite la materialización de una obra.

- Valor histórico: es una fuente de información, pues refleja el desarrollo de la arquitectura y urbanismo en un determinado momento y lugar.

El valor legal viene dado por el proceso de visado, realizado por el colegio profesional competente, que proporciona además unas garantías mínimas de calidad.

El nacimiento de los colegios viene marcado por el Real decreto 2653, de 27 de diciembre de 1929, por el que se hace condición obligatoria para el ejercicio de la profesión de arquitecto en España, a partir del 1 de marzo de 1930, pertenecer a un colegio de arquitectos.

La necesidad de modernización marca la redacción del Real drecreto 327/2002, de 5 de abril, por el que se aprueban los Estatutos Generales de los Colegios Oficiales de Arquitectos y su Consejo Superior (modificación del Decreto de 13 de junio de 1931).

Las funciones de los colegios oficiales de arquitectos se agrupan en las categorías de registro, represen- 
tación, ordenación, servicio y organización. En ésta se explicita la función de "visar los trabajos profesionales de los arquitectos con el alcance dispuesto por las normas estatutarias, las corporativas y las leyes".

Son objeto del visado colegial los trabajos profesionales que se reflejen documentalmente y estén autorizados con la firma del arquitecto.

El visado permite así acreditar la identidad del arquitecto responsable (y por tanto su habilitación) y comprobar la integridad formal de la documentación presentada.

En la actualidad, los distintos colegios, tanto de arquitectos como de ingenieros, están implantando el llamado visado electrónico: los proyectos en formato pdf son subidos a una web, desde donde el colegio correspondiente lo descarga, visa mediante marca de agua y devuelve al autor, con todas las garantías legales, facilitando así la gestión del proceso.

Desde el punto de vista documental este tipo de visado influye en los soportes usados y en los campos de descripción, pero no en la estructura conceptual del proyecto, aspecto en el que se centra este trabajo.

\section{Caracterización de un proyecto arquitectónico}

Las tipos de proyectos vienen dados por el Real decreto 2512/1977, aunque cada colegio lo ha adaptado de forma diferente, generando evidentes dificultades de normalización.

Se propone la caracterización de un proyecto en función de cuatro aspectos: clase/subclase, uso, modalidad y fase/etapa.

\subsection{Clase/subclase}

Sirve para tipificar los distintos proyectos susceptibles de ser visados (y tarifados), en función del tipo de actividad que se realiza.

La tabla 1 muestra las clases y subclases de proyectos, según el citado Real decreto 2512/1977.

En el caso de los proyectos de edificación, la subdivisión se basa en Uniclass, por ser una taxonomía estandarizada y que se acopla perfectamente a los usos.

\subsection{Uso}

Se crean a partir de la taxonomización de las clases de trabajos de edificación (aunque se pueden aplicar al resto de clases) y sirven para identificar la función a la que va a estar destinada la obra.

En Uniclass se puede consultar un listado exhaustivo de usos. En la tabla 2 se muestra, a modo de ejemplo, un listado selectivo de éstos, traducidos y adaptados.

\begin{tabular}{|l|l}
\hline CLASE & SUBCLASE \\
\hline
\end{tabular}

A. Edificación
A.1. Instalaciones de ingeniería civil. Servicios.
A.2. Instalaciones industriales
A.3. Instalaciones administrativas
A.4. Instalaciones comerciales
A.5. Instalaciones de servicios de protección
A.6. Instalaciones sanitarias, de salud y bienestar
A.7. Instalaciones de recreo
A.8. Instalaciones religiosas
A.9. Instalaciones educativas, científicas y de información
A.10. Instalaciones residenciales
A.11. Otras instalaciones

\section{B. Urbanismo \\ B.1. Planes generales municipales de} ordenación

B.2. Planes parciales

B.3. Estudios de detalle. Ordenación de volúmenes

B.4. Proyectos de urbanización y de obras públicas

B.5. Programas de actuación urbanística

B.6. Planes especiales

B.7. Normas subsidiarias de planeamiento

B.8. Normas complementarias de planeamiento

B.9. Proyectos de delimitación de suelo urbano

B.10. Proyectos de parcelación

B.11. Proyectos de reparcelación

B.12. Proyectos de expropiación

B.13. Otros trabajos de urbanismo y planeamiento

B.14. Asesoramiento urbanístico

C. Deslindes

\section{Replanteos}

E. Mediciones

E.1. De terrenos

E.2. De solares

E.3. De edificaciones

\section{F. Tasaciones}

F.1. De terrenos y solares

F.2. Tasaciones complementarias del suelo

F.3. Tasación total de edificios

F.4. Tasación de las rentas

F.5. Tasación de derechos reales

F.6. Varios

G. Reconocimientos

\section{H. Examen de documentos}

I. Consultas

J. Diligencias

K. Informes

L. Dictámenes

M. Peritaciones

N. Actas y certificados

N.1. Certificado parcial de fin de obra

N.2. Certificado final de fin de obra

N.3. Acta de recepción final de obra

N.4. Otros certificados

\section{O. Arbitrajes}

P. Otros

Tabla 1. Clases y subclases de proyectos arquitectónicos (fuente: Real decreto 2512/1977 y Uniclass) 


\begin{tabular}{|c|c|}
\hline CódIGO & $\begin{array}{c}\text { USO } \\
\text { Instalaciones educativas, científicas } \\
y \text { de información }\end{array}$ \\
\hline .001 & Academias de arte y diseño \\
\hline .002 & Academias de música, cine, danza \\
\hline .003 & Academias técnicas, politécnicas \\
\hline .004 & Academias de educación \\
\hline .005 & Archivos y oficinas de patentes \\
\hline .006 & Biblioteca escolar \\
\hline .007 & Biblioteca especial \\
\hline .008 & Biblioteca nacional \\
\hline .009 & Biblioteca pública \\
\hline .010 & Biblioteca universitaria \\
\hline .011 & Centro de consulta \\
\hline .012 & Educación primaria \\
\hline .013 & Educación secundaria \\
\hline .014 & Escuela de enfermería \\
\hline .015 & Estudio de arte, oficinas de dibujo \\
\hline .016 & Instalaciones científicas \\
\hline .017 & Instalaciones de información, partes \\
\hline .018 & Instalaciones educativas para adultos \\
\hline .019 & Museos, galerías de arte, planetarios \\
\hline .020 & Observatorios \\
\hline .021 & Otras \\
\hline .022 & Para grupos especiales \\
\hline .023 & Partes \\
\hline .024 & Salas de instrumentación \\
\hline .025 & Universidades \\
\hline
\end{tabular}

Tabla 2. Usos: Instalaciones educativas, científicas y de información (fuente: Uniclass)

\subsection{Modalidad}

Sirve para facetar el tipo de trabajo que se realiza en un lugar destinado a un determinado uso.

\subsection{Fase/etapa}

Una obra se realiza en diferentes fases (independientes a efectos de visado y tarifación), de forma que cada una comprenda a las anteriores y represente un mayor grado de definición del trabajo.

Además de las fases marcadas por el Real decreto 2512/1977, se consideran las siguientes:

- Concurso: propuesta (equivalente a un anteproyecto o a un análisis funcional) que se presenta a evaluación, donde sólo en caso de ganar se procede a la realización completa del proyecto.

- Proyecto de final de obra: documentación que recoge la obra ejecutada e incorpora todas las modificaciones realizadas en ella a lo largo del proceso de ejecución (Ley 3/2004, Comunidad Valenciana).

Las distintas fases de un proyecto se muestran en la tabla 4. Un análisis completo de las mismas se puede consultar en Carrascal Simón (1994).

Finalmente, se plantea el concepto de etapa, que identifica la realización de una parte de la obra en una determinada fase del trabajo, y que implica un nuevo visado.

\section{Composición de un proyecto de arquitectura}

Los cinco componentes de un proyecto son la memoria, planos, pliego de condiciones, mediciones y presupuesto. Son las unidades mínimas independientes de un proyecto, y todas llevan la firma del arquitecto a modo de colofón.

Vienen determinados por el Código Técnico de la Edificación.

Desde la web del Consejo Superior de los Colegios de Arquitectos de España (Cscae), se puede acceder a la normativa de visado y presentación de trabajos de todos los colegios territoriales. En ella se detallan los componentes obligatorios (y su contenido) para el visado, en función de la clase y fase del proyecto.

\section{http://www.cscae.com/consejo/consejo00.html}

\begin{tabular}{|c|l|}
\hline CóDIGO & \multicolumn{1}{|c|}{ MODALIDAD } \\
\hline 01 & Ampliación \\
\hline 02 & Apuntalamiento \\
\hline 03 & Avance \\
\hline 04 & Decoración \\
\hline 05 & Derribo \\
\hline 06 & Elevación \\
\hline 07 & Estudio de impacto ambiental \\
\hline 08 & Jardinería y paisaje \\
\hline 09 & Legalización \\
\hline 10 & Modificación \\
\hline 11 & Obra nueva \\
\hline 12 & Planeamiento \\
\hline 13 & Reforma \\
\hline 14 & Rehabilitación: edificio \\
\hline 15 & Restauración: fachadas \\
\hline & \\
\hline
\end{tabular}

Tabla 3. Modalidad de trabajos (fuente: Coacv)

\begin{tabular}{|c|l|}
\hline CóDIGO & FASE \\
\hline 01 & Concurso \\
\hline 02 & Estudio previo \\
\hline 03 & Anteproyecto \\
\hline 04 & Proyecto básico \\
\hline 05 & Proyecto de ejecución \\
\hline 06 & Dirección de obra \\
\hline 07 & Proyecto final de obra \\
\hline 08 & Liquidación y recepción de obra \\
\hline
\end{tabular}

Tabla 4. Fases de un proyecto 


\section{Proyectos parciales}

Son proyectos parciales, según la Ley 38/1999, de 5 de noviembre, de Ordenación de la Edificación, aquellos referidos a "tecnologías específicas o instalaciones del edificio que no se hayan incorporado al proyecto de edificación, bien por su complejidad o bien por no ser competencia del autor del mismo".

A continuación se indican los proyectos parciales obligatorios:

- Infraestructuras comunes de telecomunicación.

- Estudio de seguridad y salud laboral, básico o no.

- Urbanización de parcela o ámbito vial.

- Actividad.

- Instalación de ascensor.

- Abastecimiento de agua.

- Instalación eléctrica.

El resto de los proyectos parciales son opcionales, a decidir por el promotor. Entre ellos destacan los de instalación de gas, climatización, calefacción, sistemas audiovisuales e informáticos, y otros equipamientos, como piscinas, pistas deportivas, etc.

\section{Documentación asociada}

Se trata de material de naturaleza administrativa, que no forma parte de los trabajos reseñados en la tabla 1.

Se destaca la siguiente documentación:

- Acta de replanteo, justificativo del inicio de las obras.

- Licencia de obras y otras autorizaciones administrativas.

- Libro de órdenes y asistencias.

- Libro de incidencias en materia de seguridad y salud.

- Libro de gestión de calidad de obra.

- Libro del edificio.

- Bocetos, maquetas y cualquier otro material de apoyo.

\section{Propuesta de diseño conceptual}

Se propone el siguiente esquema conceptual, basado en un modelo entidad-relación (E-R), para tratar los proyectos visados arquitectónicos (figura 1).

Un primer grupo de entidades, en color azul, caracteriza al proyecto desde un punto de vista documental.

En un nivel superior se establece la entidad "obra", meramente conceptual, que marca el inicio de una se- rie. De ella depende directamente la entidad "proyecto", que identifica al documento visado.

En un nivel inferior se encuentran los componentes (memorias, planos, etc.). También se refleja la posibilidad de que éstos puedan ser modificados y, por tanto, nuevamente visados.

Como documentación relacionada se consignan los proyectos parciales (relacionados con la entidad "proyecto"), y la documentación asociada (relacionada con "obra").

Un segundo grupo de entidades, marcado en gris, hace referencia a la caracterización de los documentos (clase, subclase, uso, modalidad y fase).

El tercer grupo de entidades, marcado en verde, hace referencia al soporte de los documentos. La entidad superior es "soporte", y es meramente conceptual.

En este caso, cada documento, de forma disjunta, queda almacenado en el servidor (memoria interna), en una memoria externa ( $\mathrm{dvd}$ o memorias flash preferentemente) o en papel (los proyectos se encuadernan formando tomos, que se agrupan en cajas).

Tanto "obra" como "cliente" se relacionan con la entidad "lugar", que determina tanto el lugar donde se realiza la obra como la procedencia del cliente.

Este esquema sólo presenta una propuesta de modelo, centrada en los proyectos visados, con el objetivo de caracterizarlos documentalmente. Para obtener una base de datos completa, debería ampliarse con otros módulos de información: datos del arquitecto, de gestión, etc.

Así mismo, la entidad "componentes" debería especializarse para poder describir sus partes independientemente. Esto sería necesario en el caso de los planos.

La mayor o menor granularidad en la especialización y atributos deberá estudiarse en cada caso. La descripción exhaustiva de cada plano tiene sentido en el archivo de un colegio profesional, pero no es viable en un estudio de arquitectura.

Finalmente, la implantación del visado digital, comentada anteriormente, debería influir en el desarrollo de nuevos atributos (campos de descripción), pero no de nuevas entidades.

\section{Propuesta de código Inpav}

Se propone el código de identificación Inpav (Identificación nacional de proyectos de arquitectura visados), el cual se compone de cuatro elementos, separados por guión.

El primero se refiere a la obra, el segundo a las características del proyecto (clase, subclase y uso), el tercero designa la modalidad de proyecto y el cuarto la fase de la obra. 


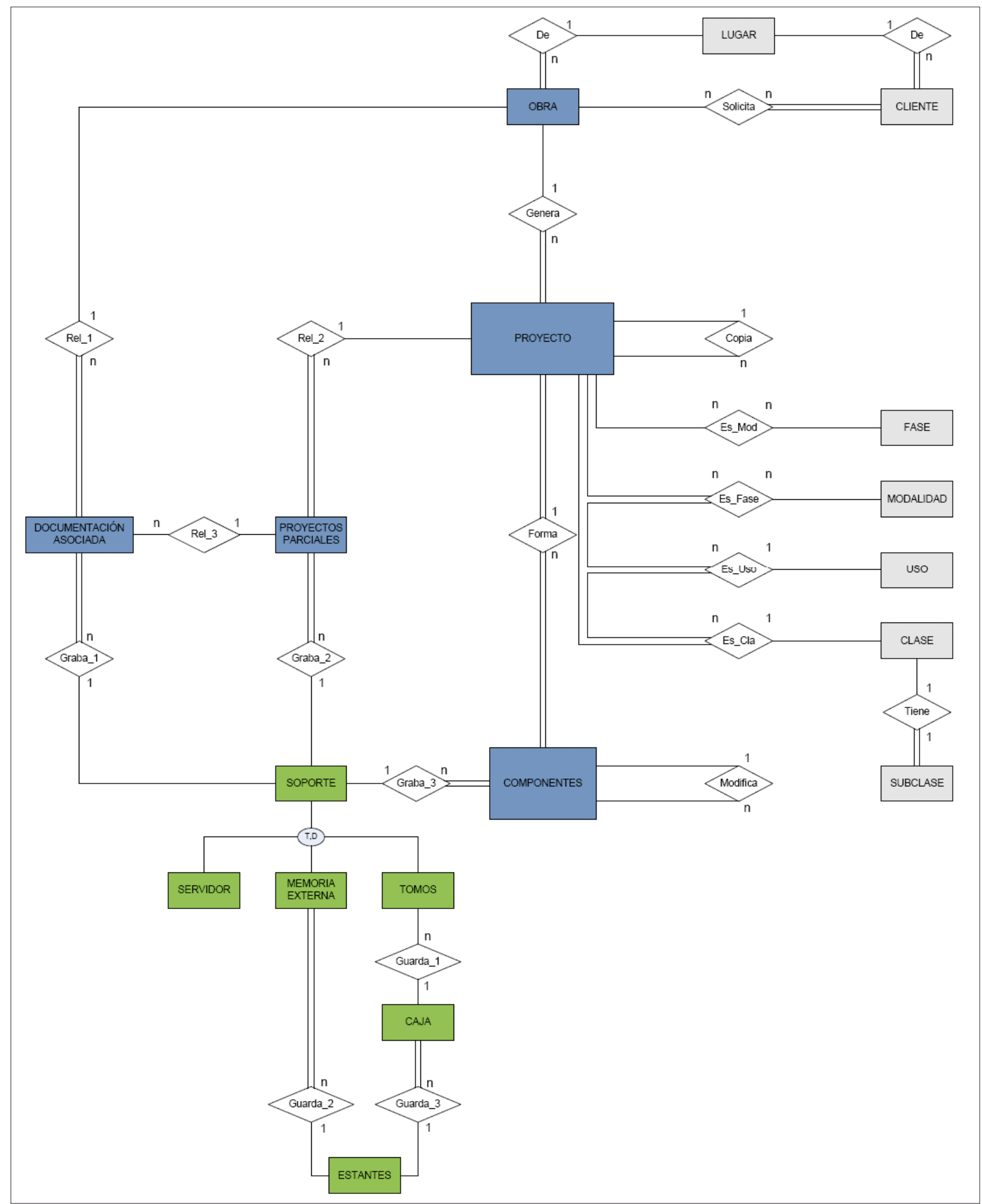

Figura 1. Esquema conceptual para proyectos visados arquitectónicos

El primer elemento está compuesto por cuatro subelementos, separados por el símbolo (/):

- Código Nuts (Nomenclature of Units for Territorial Statistics, o Nomenclatura de áreas para realizar estadísticas territoriales) de nivel 3 (provincias en el caso de España), para señalar el territorio donde se eje- cuta la obra. Este código comienza con las dos letras del código ISO 3166-1, al que se le añaden tres cifras relativas a la comunidad autónoma (Nuts 2) y la provincia (Nuts 3), total 5 cifras.

- Cinco cifras, que indican el estudio o arquitecto que realiza el proyecto. 
- Número de obra que dicho estudio o arquitecto ha visado en el año (3 cifras).

- Fecha de visado del proyecto (día_mes_año).

El segundo elemento está formado por tres subelementos, separados por el símbolo (.):

- Una letra, para la clase de proyecto.

- Dos cifras, de la subclase.

- Tres cifras, que identifican el uso.

En el caso de que la clase de proyecto no tuviese subclases, el número de la subclase sería " 00 ".

\section{"El código Inpav puede ser útil para la identificación y recuperación de proyectos"}

El tercer elemento queda formado por dos cifras que indican la modalidad de trabajo. En el caso de coexistir más de una, se procederá a unir mediante el símbolo (/) los códigos correspondientes, de la forma: $\mathrm{xx} / \mathrm{xx} / \ldots$

El cuarto elemento queda igualmente formado por dos cifras que identifican la fase de la obra. En caso de coexistir más de una fase, se procederá a unir mediante el símbolo (/) los códigos correspondientes de la forma: $\mathrm{xx} / \mathrm{xx} / \ldots$

En el caso de que se tratase de un proyecto al que no fuese aplicable el concepto de fase, se marcaría todo el elemento mediante " $\mathrm{X}$ ".

La fase quedaría perfilada a través de la etapa, mediante una cifra precedida de "//". En caso de no existir etapas parciales, se dejaría en blanco.

La forma final del Inpav es la siguiente:

Inpav: $\mathrm{xxxxx/xxxxx/xxx/xx \_ xx \_ xxxx} \mathrm{-} \mathrm{Letra.xx.xxx} \mathrm{-}$ $\mathrm{xx} / \ldots-\mathrm{xx} / \ldots / / \mathrm{x}$

Según la codificación usada, un proyecto de ejecución de un instituto en Valencia, donde se realiza una restauración, quedaría identificado de la siguiente forma:

Inpav: ES523/00001/001/19_08_2009 - A.9.013 - 15 $-05 / /$

\section{Conclusiones}

- Los proyectos visados se pueden caracterizar documentalmente. No obstante, la legislación y normativa existente está dispersa y es confusa, siendo necesaria la redacción de una disposición y/o norma que regule mejor este aspecto.
- El proyecto visado se puede analizar en dos niveles: según su tipo (clase/subclase, uso, modalidad y fase/etapa) y según su composición (memoria, planos, pliego de condiciones, mediciones y presupuesto).

- Debido a las diferencias en la regulación de la profesión en diferentes países, no es posible una normalización del proyecto en el ámbito internacional.

- El modelo conceptual propuesto pretende ser la base para la construcción de un sistema de recuperación de proyectos y, en ningún caso, para su ordenación física.

- La especificación de los atributos del modelo y su normalización, serían el siguiente paso hacia la confección de unas reglas de catalogación adecuadas a estos materiales.

- El Inpav, revisado y refinado, puede ser una herramienta útil para la identificación y recuperación de proyectos. Esto precisará de un desarrollo exhaustivo y con carácter normativo de clases, subclases, usos, modalidades y fases, donde la aplicación de lenguajes normalizados como Uniclass será recomendable.

- Sería interesante la ampliación de este estudio a otras áreas profesionales reguladas por colegios, como la industrial o las telecomunicaciones.

\section{Agradecimientos}

A todo el equipo de Áreas, Ingeniería y Arquitectura, estudio del arquitecto José-María Tomàs-Llavador.

\section{Referencias}

Blanco, Manuel. "Architectural archives: from collection to integration". En: $15^{\text {th }}$ Intl. congress on archives 2004, 23-29 August, Viena, Austria. Archivos, memoria y conocimiento.

http://www.wien2004.ica.org/imagesUpload/pres_39_BLANCO_SAR01. pdf?PHPSESSID=9a735b20bad3217a2970ede72f14dbd6

Carrascal-Simón, Andreu. "Archivos profesionales de arquitectura. Estudio de una serie y su tipología documental: el proyecto de construcción". Lligall, 1994, v. 8, pp. 113-123.

Carrascal-Simón, Andreu; Gil-Tort, Rosa. Los documentos de arquitectura y cartografía: qué son y cómo se tratan. Gijón: Trea, 2008.

Consejo superior de los colegios de arquitectos de España (Cscae). http://www.cscae.com/

Cox, Richard J. "The archival documentation strategy and its implications for the appraisal of architectural records". American archivist, June 1996, v. 59 , n. 2, pp. 144-155.

Ekholm, Anders. "A conceptual framework for classification of construction works", March 1996, ITCon, v. 1.

http://www.itcon.org/1996/2/paper.htm

Eurostat. Regiones: Nomenclatura de unidades para realizar estadísticas territoriales -Nuts-, mayo de 1999.

http://epp.eurostat.ec.europa.eu/cache/ITY_OFFPUB/CA-22-99-442-1F/ EN/CA-22-99-442-1F-EN.PDF

Hildesheimer, Françoise. "El tratamiento de los archivos de arquitectos: el caso de Francia". ONU: Paris, 1987.

Ley 2/1974, de 13 de febrero, sobre Colegios Profesionales. 
Ley 3/2004, de 30 de junio, de Ordenación y fomento de la calidad de la edificación. Comunidad Valenciana.

Ley 38/1999, de 5 de noviembre, de Ordenación de la edificación.

Ley 7/1997, de 14 de abril, de Medidas liberalizadoras en materia de suelo y de colegios profesionales.

Maygene, Daniels. "Description of architectural records". En: A guide to the archival care of architectural records 19th-20th centuries. Paris: ICA, 2000 .

Nyambayo, James; Faraj, Ihsan; Amor, Robert. "Product libraries-technology review". En: Proceedings of the 3rd European conference on product and process modelling in the building industry EC-PPM'2000, Lisbon, Portugal, 25-27 September, pp. 267-274.

Real decreto 2512/1977, de 17 de junio, por el que se aprueban las tarifas de honorarios de los arquitectos en trabajos de su profesión.

Real decreto 2653. En: Gaceta de Madrid, 28 de diciembre, 1929, n. 362.

Real decreto 314/2006 de 17 de marzo por el que se aprueba el Código técnico de la edificación.
Real decreto 327/2002 de 5 de abril por el que se aprueban los Estatutos generales de los Colegios Oficiales de Arquitectos y su Consejo Superior.

Real decreto 523/2005 de 13 de mayo, por el que se modifican los Estatutos Generales de los Colegios Oficiales de Arquitectos y su Consejo Superior, aprobados por el Real decreto 327/2002, de 5 de abril.

Uniclass: unified classification for the construction industry. London: Riba publishing, 1997.

http://www.ribabookshops.com/site/viewtitle.asp?pid $=982$

Section on architectural records. A guide to the archival care of architectural records 19th-20th centuries. Paris: ICA, 2000.

Xiaomi, An. "On integration best practice of architectural project archives". Records Management Society Bulletin, Feb. 2006, n. 130, pp. 33-38.

Enrique Orduña-Malea, Universidad Politécnica de Valencia

enorma@fiv.upv.es

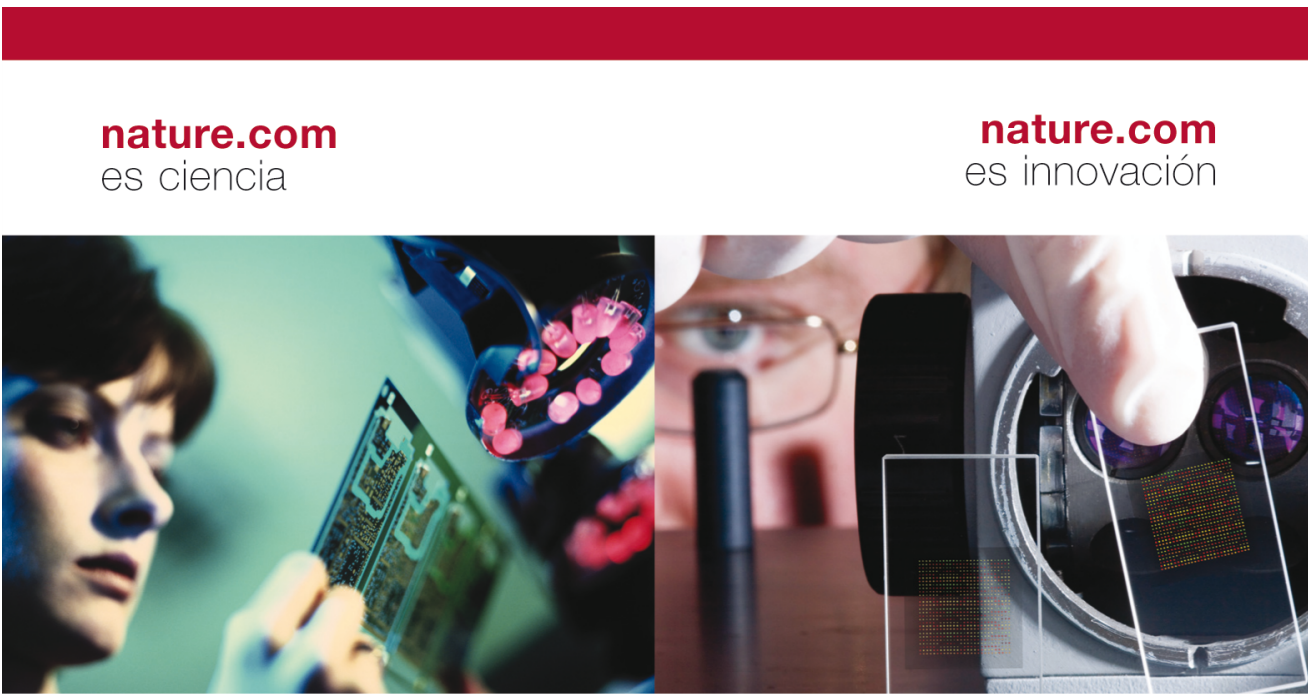

\section{Todo está en nuestro nature com}

Consiga acceso a nature.com con una licencia de sitio de Nature Publishing Group (NPG) y elija entre las revistas de investigación científica y médica de más alta calidad. Desde la distribución de documentos hasta la evaluación de artículos por expertos, nature.com ofrece numerosas aplicaciones emprendedoras en Web 2.0, entre las cuales encontrará:

- blogs como Nature Newsblog

- RSS feeds

- podcasts

- videos
- referencia de documentos con Connotea

- redes sociales en Nature Network

- Nature Precedings, un foro que permite compartir trabajos de investigación antes de publicación

- organizar y compartir datos con Scintilla

Recuerde - el acceso en línea a las publicaciones de Palgrave Macmillan está disponible con una licencia de sitio a través de NPG.

T: +44 (0) 2078434759 | E: institutions@nature.com | W: www.nature.com/libraries 

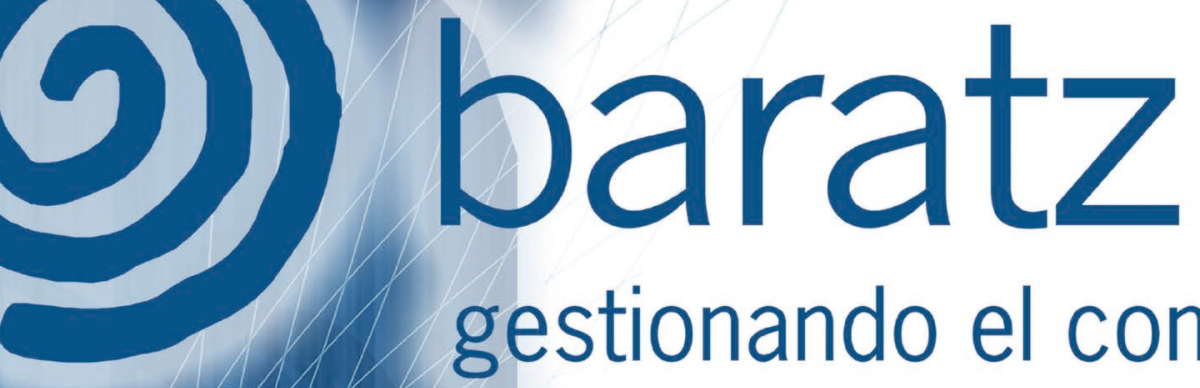

\section{gestionando el conocimiento}

\section{Soluciones integrales} para la gestión de:

(2) Bibliotecas

(9) Archivos

(9) Contenidos empresariales

Tel: 34914560360 informa@baratz.es www.baratz.es 